\title{
Derrière la langue, les positionnements sociaux. Pouvoir faire entendre sa voix en langue seconde.
}

\author{
Malika Ben Harrat \\ Anne-Christel Zeiter \\ Université de Lausanne \\ Suisse
}

\begin{abstract}
L'éducation a beau être, de droit, l'instrument grâce auquel tout individu, dans une société comme la nôtre, peut avoir accès à n'importe quel type de discours, on sait bien qu'elle suit dans sa distribution, dans ce qu'elle permet et dans ce qu'elle empêche, les lignes qui sont marquées par les distances, les oppositions et les luttes sociales. Tout système d'éducation est une manière politique de maintenir ou de modifier l'appropriation des discours, avec les savoirs et les pouvoirs qu'ils emportent avec eux.
\end{abstract}

(Foucault, 1970, p. 244)

Afshin ${ }^{1}$, un architecte iranien d'environ 40 ans, se présente à une épreuve orale de français. La tâche demandée est destinée à faire décrire une routine, et l'examinatrice l'interroge donc sur le déroulement d'une journée habituelle lorsqu'il travaillait en Iran. L'homme décrit alors une matinée type : il consulte les plans, se rend sur les chantiers pour contrôler les travaux, rencontre différents corps de métiers et rentre chez lui pour manger. II s'arrête là. Après une hésitation, l'examinatrice le relance pour savoir ce qu'il fait l'après-midi, ce qui semble le surprendre. II lui répond, l'air un peu impatienté, que « après-midi même chose . comme le fini le bâtiment . le même chose ((!)) ". Afshin accompagne son discours de mouvements de mains calmes, ses avant-bras restant toujours sur la table devant lui. II parle beaucoup et semble à son aise, en particulier lorsqu'il s'agit de son travail, au point que cette question sur son emploi du temps parait effectivement incongrue. À bien y réfléchir, dans quelle situation demanderait-on à un architecte le détail de ses journées? Afshin le souligne par la légère ironie de sa réponse : tant qu'un bâtiment n'est pas terminé, l'architecte navigue entre ses plans, les ingénieurs et le chantier, il n'y a pas grand-chose de plus à en dire. L'entretien se poursuit donc sur un autre sujet.

Au moment d'évaluer le niveau de langue d'Afshin, les experts soulignent sa capacité à défendre son point de vue, à exprimer sa pensée dans n'importe quelle situation ou presque en français, et même à faire preuve d'ironie : dans une perspective actionnelle, il remplit donc les critères d'une compétence intermédiaire (B1 CECR). Pourtant, comme en témoigne l'extrait ci-dessus, peu de choses fonctionnent dans son parler au niveau morpho-syntaxique, ce qui le situe à un niveau débutant (A1+CECR). Sa maitrise effective du français en tant que système linguistique est ainsi en fort décalage avec sa capacité à occuper la place comme homme et architecte, en tout cas dans ce contexte spécifique d'une évaluation de langue: la migration semble n'avoir impacté que sa langue et rien d'autre. Or, lorsque l'on consulte son dossier, on s'aperçoit qu'il n'en est rien : Afshin enchaine les mesures de réinsertion professionnelle, et donc les cours de langue, depuis plus d'une année, et il n'a pas encore trouvé de travail. Une chose frappe, néanmoins : si, à l'oral du moins, il semble avoir fossilisé ses ressources à un niveau lui permettant de se faire comprendre, sa capacité à produire l'image d'un homme de haut niveau, sûr de lui et volontiers ironique comble en partie ses lacunes langagières, ce qui n'est pas sans nous questionner sur ce que signifie apprendre une nouvelle langue.

Ces situations, courantes dans le monde de l'enseignement-apprentissage des langues, ne cessent d'interroger. Certaines personnes n'osent pas s'exprimer tant que leur maitrise du système ne leur semble pas parfaite, et lorsqu'elles le font, elles savent bien peu mettre leurs compétences en valeur. D'autres au contraire savent faire de trois bouts de ficelle linguistiques un discours persuasif et donnent l'impression que la langue n'est pas un enjeu. Et entre ces deux extrêmes, toutes sortes de voix se font entendre. Sur la base de ce constat, nous nous intéressons ici à la voix de la personne sur deux plans. D'une part, le sens qu'elle donne à ses expériences sociales et langagières est susceptible d'influencer le développement de son répertoire langagier, ce qui se manifeste dans son discours par le recours (1) à divers positionnements plus directement liés à l'interaction en cours, et (2) à des représentations sociales destinées à argumenter, justifier ou expliciter ses positions (Grize, 1990). D'autre part, la manière dont la personne développe sa compétence à se positionner socialement en mobilisant les pans de son répertoire langagier acquis dans la nouvelle langue lui permet de pallier des lacunes plus directement linguistiques et donc de mener à bien les agir sociaux dans lesquels elle est engagée. La voix ainsi pensée n'est évidemment pas uniquement caractéristique des expériences langagières en L2. Toutefois, dans un tel contexte, il s'agit d'une compétence à réapprendre: la déqualification sociale et professionnelle si souvent liée à la migration implique de reconfigurer sa place sociale, ce qui est loin d'être évident lorsque la langue empêche de faire montre de ses compétences sociales et professionnelles et s'ajoute à d'autres marques d'altérité potentiellement discriminantes. Or, nous posons l'hypothèse que cette difficulté avant tout sociale peut se travailler, avec des personnes apprenantes, dans des cours de langue, par une approche plus proprement linguistique et par un encouragement à faire preuve de réflexivité sur les expériences sociales et langagières 
en L2 (Jeanneret, 2010). Les théories de la socialisation langagière (Dubar, 2010 ; Duff, 2003 ; Roberts, 1998 ; entre autres) mettent en effet en évidence le rôle fondamental joué par la possibilité de faire entendre sa voix dans l'accès aux pratiques sociales, des pratiques que les perspectives plaçant l'agir social au cœur de l'apprentissage considèrent comme déterminantes (Busch 2013, 2015 ; Blommaert et Backus, 2012).

Dans ce contexte, la notion de voix recouvre donc à la fois la voix que la personne a et celle qu'on lui accorde, ce qui tend à voiler nombre de processus. C'est en ce sens que nous proposerons ici de la penser en termes de positionnements de différents ordres, chacun représentant une manière de prendre sa place ou d'être mis à une certaine place dans l'interaction. Cette idée sera investiguée sur la base d'extraits d'entretiens de recherche menés avec des apprenant·e-s adultes de niveau débutant-intermédiaire (A2-B1 CECR) et recueillis dans le cadre de la thèse de doctorat de Malika Ben Harrat (en cours), qui poursuit notamment les réflexions menées à l'Université de Lausanne sur la biographie langagière ${ }^{2}$. Après avoir brièvement dressé le cadre théorique qui sous-tend notre réflexion, nous proposerons une définition de la voix comme positionnement social et langagier en prolongement de Davies \& Harré (1990). En les illustrant d'extraits d'entretiens (corpus Ben Harrat), nous identifierons trois ordres de positionnements sociaux discursifs (Van Langenhove et Harré, 1999, pp. 20-21) qui rendent compte des dynamiques interactionnelles et des relations de pouvoir qui s'y matérialisent.

\section{De l'agir social au répertoire langagier : pouvoir et réflexivité}

La perspective adoptée ici considère l'agir social comme ingrédient fondamental pour l'appropriation d'une L2, ce qui implique de s'intéresser à ce que signifie agir et se socialiser dans un nouveau contexte social et langagier. Autrement dit, la conscience des expériences sociales effectives et du sens que la personne ellemême leur donne s'avère essentielle. Comme répertoire langagier (Busch, 2015), la langue représente en effet ici l'ensemble des ressources énonciatives et communicatives de la personne en différentes langues et dans toutes ses variations, des ressources construites en fonction des interactions auxquelles cette dernière participe et des rôles sociaux qu'elle s'attribue et se voit attribuer, dans une perspective sociohistorique. Busch (2015) souligne par ailleurs l'importance des facteurs psychiques, physiques et affectifs qui, de manière dynamique avec des facteurs interactionnels et historico-politiques régulent les expériences sociolangagières. Or ces expériences ne sont jamais neutres, et la personne leur donne sens en recourant aux représentations sociales (Jodelet, 1989, 2008 ; Zeiter, 2018) qu'elle a construites jusque-là, sur la base d'événements passés, de préoccupations actuelles et de projections vers l'avenir, donc de sa socialisation. Selon Jeanneret (2010), le développement du répertoire langagier se fait ainsi par et dans l'agir social et permet, en retour, lorsque tout se passe bien (et vice-versa !) d'agir de manière plus adéquate grâce à de nouvelles ressources.

Les ressources langagières ainsi construites peuvent être distinguées, dans une visée analytique, en trois types de compétences qui concernent (1) le système à proprement parler (morpho-syntaxe, lexique, phonologie, etc.), dont la maîtrise renforce la correction linguistique et donc la précision langagière, (2) les normes pragmatiques, fondamentales pour éviter les malentendus et autres blocages communicatifs et, enfin, (3) les positionnements dans le champ du pouvoir, essentiels pour mener à bien ses objectifs. Ce troisième pan du répertoire langagier est d'importance, dans la mesure où une langue marquée d'altérité peut, en interaction avec d'autres marqueurs sociaux comme le genre, l'âge, l'ethnicité, etc., provoquer le rejet autant que l'appartenance à une communauté donnée et structure donc les modes de participation de la personne à l'espace social. La capacité à mieux se positionner dans le cadre de situations interactionnelles et sociales délicates peut alors, comme chez Afshin, pallier d'autres manques et permettre d'établir la communication, soit d'agir socialement. En ce sens, dans le contexte de cours de langue, Jeanneret (2010) insiste sur l'importance d'une culture institutionnelle destinée à développer la réflexivité des apprenant $\cdot$ - $s$ de langue sur leurs pratiques sociales et langagières, et donc sur les enjeux subjectifs et sociaux qui structurent leur entrée dans la langue. S'il s'agit en effet d'identifier le(s) rôle(s) que jouent la langue et son appropriation dans les pratiques sociales de l'individu pour mieux saisir la manière dont ce dernier peut plus ou moins y accéder (Zeiter, 2019), nous considérons que la mise en discours d'une représentation permet d'accéder au(x) sens accordé(s) par une personne à des objets et des individus, sens qui influe(nt) fortement sur ses expériences sociales effectives. En retour, les représentations sociales sont donc des observables qui renseignent également sur les positionnements discursifs individuels, des positionnements qui s'avèrent essentiels à la socialisation et donc à l'apprentissage d'une nouvelle langue. Accéder à de tels observables est donc précieux pour la recherche en sciences humaines, évidemment, mais également pour l'enseignement des langues, qui doit en tenir compte.

\section{Trois ordres de positionnements sociaux}

Dans ce cadre, il faut considérer que les enjeux de pouvoir auxquels se confrontent les individus se matérialisent, discursivement, par des représentations et des positionnements sociaux intrinsèquement liés. Les positionnements sociaux prennent en effet appui sur des représentations sociales qui à la fois précèdent 
les interactions, s'y enrichissent ou s'y cristallisent. Ces représentations énoncent la perception d'un individu (Dortier, 2008) sur un objet donné et renseignent sur ses rapports au monde (Jodelet, 2005), donc sur ses positionnements : en d'autres termes, comment et pourquoi il occupe sa place dans différents espaces sociaux. En retour, les positionnements sociaux correspondent à l'assignation discursive positive neutre ou négative de droits, devoirs ou obligations (Kayi-Aydar, 2019) envers soi-même, c'est-à-dire qu'une personne s'attribue un droit (autopositionnement), ou envers autrui, lorsqu'une personne attribue ce droit à une autre (hétéropositionnement). En d'autres termes, on (se) positionne toujours en relation à un objet et à la manière dont on se le représente. II faut par ailleurs mentionner que la capacité à se positionner socialement varie fortement d'une personne à une autre (Harré, 2012) et selon les contextes et les langues dans lesquelles elles agissent. Sur le constat de cette différence, nous postulons que cette capacité est une compétence qui peut s'acquérir comme d'autres ressources langagières et communicatives, pour autant que le processus soit conscient.

Afin de pouvoir les didactiser pour la classe de langue, il importe toutefois dans un premier temps de comprendre finement la manière dont ces positionnements sociaux se succèdent et s'entrecroisent. Comme nous le montrerons ici, nos analyses distinguent trois ordres de positionnement conformes à la perspective de Van Langenhove et Harré (1999), dont il s'agit d'identifier les fonctions et les effets. Le positionnement de premier ordre correspond aux consignes que donnent les examinateurs à Afshin : implicitement, en la formulant, ils s'autopositionnent en tant que tels et hétéropositionnent Afshin comme candidat. Le positionnement de deuxième ordre relève d'une réponse au premier, qui peut être ambivalente. Dans le cas d'Afshin, cet hétéropositionnement s'avère d'abord performatif (Kayi-Aydar, 2019) puisqu'il l'accepte et le montre en racontant sa matinée. II le limite, toutefois, en refusant de s'exprimer inutilement et de raconter ses après-midis : ici, Afshin se repositionne plus comme architecte que comme apprenant de français, et l'on s'aperçoit que le positionnement de premier ordre n'était donc que partiellement performatif, n'étant pas complètement perçu « comme allant de soi »³ (Van Langenhove et Harré, 1999 ; Harré, 2012) par Afshin.

Enfin, les positionnements de troisième ordre représentent la narration d'une partie d'interaction passée, par exemple lorsque nous racontons ici l'interaction entre Afshin et ses examinateurs. Particulièrement visibles dans les discours qui relatent des événements discursifs, ils permettent d'accéder au sens donné aux échanges vécus par les personnes, raison pour laquelle nous insisterons plus particulièrement sur ces derniers dans nos analyses. En effet, en rapportant leurs expériences, les individus s'auto-re-positionnent et hétéro-re-positionnent leurs interlocuteurs dans un contexte interactionnel ayant précédé l'entrevue et nous proposent ainsi - notamment par le biais de représentations sociales - leur analyse des positionnements de premier et deuxième ordre vécus. Les différences de positionnement entre l'interaction initiale et l'interaction relatée sont ainsi révélatrices des enjeux de pouvoir perçus par les personnes qui nous les livrent. Du fait de sa fluidité, la notion de positionnement ainsi pensée devient un outil très opérant d'analyse des relations de pouvoir matérialisées à travers les voix des différents protagonistes, notamment lorsque les personnes mobilisent des représentations sur les droits et devoirs des individus dans un contexte donné.

\section{Données en contexte}

Les données présentées ici proviennent de deux entretiens semi-structurés menés en $2019^{4}$ avec des participant $\cdot e \cdot s$ adultes engagé.e's dans un programme universitaire de FLE (Ben Harrat). Ces entretiens faisaient suite à un cours axé sur la manière de se créer des occasions de pratiquer le français dans et hors de la classe de langue. Si les deux informatrices, Nasrin (28 ans) et Dijana (20 ans), suivent le même cursus linguistique préparatoire aux études universitaires en français, Nasrin l'a rejoint afin de faciliter son insertion professionnelle après avoir obtenu un Master dispensé en anglais dans une autre université suisse, alors que Dijana est arrivée en Suisse directement après la fin de son cursus secondaire, avec pour objectif de commencer ensuite un Bachelor.

Les extraits de ces entretiens que nous présentons ici montrent des types de positionnement rendant compte de dynamiques de pouvoir de natures diverses qui à la fois précèdent l'arrivée en classe de langue, se transforment en son sein, puis la dépassent, dans la mesure où chaque dynamique de pouvoir expérimentée détermine ou influence la participation des personnes à des interactions futures, et donc le développement de leur répertoire langagier (Busch, 2013). Notre analyse portera donc sur ces dynamiques de pouvoir qui ont trait aux différences langagières et sociales entre les participant $\cdot e \cdot s$ à ce programme, et qui influencent le développement de la voix des participantes. Concrètement, nous verrons que des représentations sociales variées entrent en résonance pour construire du sens sur le bien-fondé d'apprendre ou de pratiquer une langue ou une autre. 
Ben Harrat, Malika et Anne-Christel Zeiter. « Derrière la langue, les positionnements sociaux. Pouvoir faire entendre sa voix en langue seconde ». Nouvelle Revue Synergies Canada, № 15 (2022)

\subsection{Nasrin et sa différence}

Le premier extrait provient d'un entretien mené avec Nasrin. En se comparant à trois de ses camarades d'études, elle justifie ici pourquoi elle ne fréquente pas les autres membres du programme en dehors des cours :

\section{Exemple 1}

Ma est-ce que vous avez pu e DISCUTER avec certaines personnes e | faire des fois peut-être des activités avec certaines personnes | ((..)) déjà parlons d'aller discuter puis de boire e de boire un café à la cafétéria | par exemple

$\mathrm{Na}$ oui | oui | [...] pour e pas mal des activités | dehors des universités <ouais?> non je n'ai pas | comme ça

$((\ldots))$

Ma $[\ldots]$ vous avez la même vie que tous les autres étudiants de l'EF? ou est-ce que votre vie elle est un petit peu différente?

$\mathrm{Na}$ e je pense que | notre e vie c'était différente <ouais?> par exemple par e | quel | certaines personnes | certains étudiants sont e | seul <ouais?> se sont pas mariés <hm hm?> par exemple dans mon cours | je pense que | c'était juste MOI que mon mari était un | doctorant chez $E$. $(P)<e n$ cours de $L$ ?> oui! <d'accord ouais! ok! > e les autres sont | PARFOIS sont | très jeunes <ok> par exemple Ruzića <*ouais*> a 18 ans <ouais> plus | dix années plus jeune que moi (sourit) <d'accord> et | cette différence sont intéressants aussi | par exemple il e | ELLE e PLANIFIAIT pour e ses études e | mais parfois les autres gens sont | un petit peu âgés | ils sont juste e apprendre français pour la vie quotidienne $<{ }^{*}$ ouais ${ }^{*}>$ e parfois | ils sont $e^{*}$ comment dire $?^{*}(P)$ (réfléchit) ils sont apprendre français juste pour e apprendre une nouvelle langue $<\mathrm{hm}$ $\mathrm{hm}>$ pour e loisirs <*ah ouais*?> c'est PAS un besoin

Ma ici à l'EF ?

$\mathrm{Na}$ oui! <ah ouais!> oui! <ah!> PARFOIS il vont | il veut de cherche un travail | parfois vont de | pour poursuivre des étUdes | parfois pour des problèmes administratifs | mais je | je sais les personnes qui $<{ }^{*} \mathrm{hm}$ ? $^{*}$ e apprend français | POUR apprendre une nouvelle | chose | <d'accord | ouais >| c'est PAS un BESOIN de

Ma c'est pas un besoin | ah voilà! vous pensez à QUI par exemple? ((..)) ça m'intéresse de | d'avoir VOTRE avis

$\mathrm{Na} \quad \mathrm{hm}$ je pense par exemple | Ming par exemple | je ne sais pas que <ouais je vois ouais!> oui Ming | aussi Irina | je pense

$[\ldots]$

$\mathrm{Na}$ parce qu'il ne veut | elle ne veut PAS étudier après ça <ok> et aussi elle ne peut | ah! je ne sais pas qui il veut planifier pour chercher un travail $|<0 k>|$ tout de suite

Ma $\quad((\ldots))$ ok donc vous pensez Ming et Irina c'est pour le

$\mathrm{Na} \quad$ oui! c'était intéressant e | <ok>c'est bon | le cas de Ming c'était intéressant pour moi!

Ma ah ouais ? Pourquoi ?

$\mathrm{Na} \quad$ parce que | je | je pense que | SI j'étais à place de | d'elle <à sa place> à sa place! E je ne | e je ne choisis pas faire | comme ça

Ma vous auriez fait comment alors ? À sa place?

$\mathrm{Na} \quad$ e je ne sais pas! (rit) je ne sais pas parce que $(P)$ peut-être je préfère e $(P)$ (réfléchit) je préfère juste faire des ména | ménagers

(Entretien Nasrin, 05.06.2019, 1'15'42-1'19'14 : corpus Ben Harrat) 
Interrogée sur ses relations avec ses camarades, Nasrin justifie peu à peu sa résistance à s'adonner aux activités mentionnées, ce qui se matérialise par des positionnements comme personne différente des autres adossés à une représentation sociale liant l'âge et la trajectoire. La comparaison qu'elle établit entre elle et Ruzica sur la base d'un écart d'âge et de l'intention de cette dernière de faire des études alors qu'elle-même a déjà fini les siennes pousse Nasrin à inférer que les différences entre sa vie et celles des autres résident dans son statut de femme mariée et dans les qualifications du couple, son autopositionnement s'alignant ainsi sur l'hétéropositionnement qu'elle nous propose de son mari. Cette association de positionnements suggère un profit de distinction lié à l'apprentissage du français. On constate ainsi, à la suite de Bourdieu (2001, p. 108), que « [l]e rapport de forces linguistique n'est jamais défini par la seule relation entre les compétences linguistiques en présence. Et [que] le poids des différents agents dépend de leur capital symbolique, c'est-à-dire de la reconnaissance, institutionnalisée ou non, qu'ils reçoivent d'un groupe: l'imposition symbolique (...) ne peut fonctionner que pour autant que sont réunies des conditions sociales qui sont tout à fait extérieures à la logique proprement linguistique du discours ». À ses propres yeux, les qualifications académiques de Nasrin la distinguent des autres, qu'elle positionne implicitement - ou symboliquement - plus bas dans l'échelle sociale (comme femmes, jeunes et peu formées), justifiant ainsi de ses agir différents des leurs : elle les hétéropositionne comme n'ayant pas besoin d'apprendre le français, comme si, pour ses camarades, ce cursus était un luxe inapproprié. Pour elle en effet, étudier sans projet professionnel et sans perspective d'études ultérieures, comme elle considère que le font Irina et Ming ${ }^{5}$ semble s'apparenter à un investissement inutile : le débouché auquel elle pense lorsqu'elle se met à leur place est en effet «faire des ména | ménagers ", une activité traditionnellement féminine nécessitant peu de qualifications et peu de compétences linguistiques.

Cet hétéropositionnement de troisième ordre qui donne une voix aux protagonistes absentes en parlant (et pensant) pour elles semble expliquer son refus de prolonger les activités de la classe de langue hors de l'université par une hiérarchisation sociale : les interactions et la coopération sont obligatoires dans le cadre des activités en classe, mais le profit de distinction qu'elle énonce lui sert de justification pour ne pas côtoyer ses camarades en dehors de la classe. Ceci influence potentiellement son acquisition puisqu'elle se prive de certaines pratiques moins formelles en français, sans qu'on puisse savoir, à partir de ces données, jusqu'à quel point : il faudrait d'autres observables, et notamment des indications concernant ses pratiques sociales en français dans d'autres contextes.

\subsection{Dijana et la norme}

L'entretien mené avec Dijana, 20 ans et au tout début de son cursus universitaire, se passe principalement en français et anglais. Les extraits présentés ici soulignent, dans différents positionnements, une insécurité linguistique (Remysen, 2018) renforcée par la présence de l'enquêtrice, incarnation de diverses normes car, plus âgée, plus formée, francophone native, elle est également son ancienne enseignante de français et une spécialiste de l'acquisition des langues.

Environ trente minutes après le début de l'entretien, et bien que l'enquêtrice (Ma) n'ait donné aucune consigne proscrivant le recours à d'autres langues que le français, Dijana produit un rire et déclare avoir trop parlé en anglais ("I'm going too much in English!"), énonçant ainsi une représentation de la langue dans laquelle l'entretien devrait selon elle se dérouler. À première vue, on pourrait postuler qu'elle se positionne ici en fonction de la représentation qu'elle se fait des attentes de l'enquêtrice. Une heure plus tard, toutefois, elle évoque directement de l'insécurité :

\section{Exemple 2}

Ma est-ce que tu as confiance en toi en français ?

Di non pas du tout! je suis pas du tout à l'aise ou confiante | non pas du tout!

Ma ouais? MAIS | pourtant | jusqu'à maintenant | toutes les choses que tu m'as dites en français | je les ai compris (Dijana rit d'un air gêné) il me semble que | aujourd'hui c'est la première fois qu'on parle anglais | [...] ce que j'aimerais comprendre c'est | qu'est-ce qui FAIT que tu | ne te sens pas encore à l'aise en français? est-ce que tu peux expliquer ça?

Di oui je peux! très bien <ouah!> le problème c'est | la prononciation <hm hm?> c'est tellement $(P)$ (en soupirant) difflcile *pour moi!* non! c'est | c'est pas juste pour moi | c'est difficile

Ma est-ce que tu penses que tu as des soucis de prononciation | TOI ?

Di oui | OUI OUI 
Ben Harrat, Malika et Anne-Christel Zeiter. « Derrière la langue, les positionnements sociaux. Pouvoir faire entendre sa voix en langue seconde ». Nouvelle Revue Synergies Canada, № 15 (2022)

$\mathrm{Ma}$

TOI | si tu dois | si tu dois te

Di

oui | parce que | je t'ai déjà dit que je vois très bien les détails <ouais?> oui | c'est un problème parce que | je vois les | les détails sur mon prononciation | <AH!> sur ma prononciation

(Entretien Dijana 19.03.2019, 1'36'20-1'37'41, corpus Ben Harrat)

Face à son ancienne enseignante, Dijana déclare manquer de confiance en elle en français, de manière très générale. Lorsque l'enquêtrice l'hétéropositionne toutefois en retour comme une locutrice légitime en lui rappelant que leurs précédentes interactions en français se sont toujours bien déroulées, Dijana justifie son manque de confiance par un "problème : [...] la prononciation », difficile pour elle. Le soupir qui accompagne cette information et la transformation de sa voix en murmure renforce cette difficulté et autopositionne Dijana comme apprenante en difficulté. Elle rectifie cependant immédiatement: cette difficulté n'est pas que la sienne, ce qui laisse penser à ce stade que sa représentation de la prononciation en français comme difficile pour les allophones est communément partagée, une nouvelle norme énoncée qui la rééquilibre par rapport à ses pairs. En ajoutant cette précision, elle se repositionne au sein de ce qu'elle nous donne à voir comme une communauté de pratique (Wenger, 2009) - les apprenant·e.s de français qui ont des difficultés de prononciation - nous offrant ainsi un accès à l'une de ses représentations : parler français revient à s'approcher d'une norme de prononciation, et la norme en la matière est que ce soit difficile. L'étonnement de Ma (TOI?), qui repositionne Dijana comme locutrice légitime, ne vient pas modifier cette représentation : Dijana ajoute finalement qu'elle perçoit « les détails sur [sa] prononciation », détails qu'elle « voit très bien ». Si, globalement, elle valide ne pas avoir de problèmes de prononciation empêchant la communication, elle se positionne néanmoins à nouveau en regard de la norme qu'elle sait être enseignée dans le cursus qu'elle suit et que représente symboliquement Ma.

L'ensemble de ces éléments laissent penser que les deux interlocutrices arrivent ici à une divergence de représentations - ou impasse représentationnelle? - qui concerne à la fois les enjeux de l'appropriation langagière et les moyens d'y parvenir : bien qu'elle hétéropositionne Dijana comme une locutrice légitime, l'enquêtrice s'autopositionne également en tant que spécialiste de l'acquisition des langues. Considérant que l'informatrice ne rencontre pas de difficulté de prononciation entravant la communication, elle la pousse à questionner les raisons pour lesquelles Dijana semble faire une priorité de cet axe de la langue. Aux yeux de l'enquêtrice, cette dernière a toutes les capacités nécessaires à l'action sociale et au développement de sa voix en français. Mais du côté de Dijana, en revanche, la prononciation est donnée à voir comme primordiale car en lien direct avec son sentiment de confiance en ses capacités à produire correctement en français. Percevant un écart entre ses productions et la norme qu'elle se représente, Dijana se focalise sur cet aspect de la langue et semble non seulement en faire son objectif prioritaire, mais aussi potentiellement une condition à la participation sociale en français : elle se positionne ainsi plus comme apprenante que comme usagère de la langue. Cela laisse entrevoir l'éventualité que Dijana pourrait préférer, au moins par moments, renoncer à développer sa voix en français dans l'espace social tant qu'elle n'aura pas atteint cette adéquation à la norme qu'elle poursuit, une norme accessible de préférence lors de cours focalisés sur le système de la langue, dont notamment la prononciation, avant de la pratiquer à l'extérieur. Autrement dit, on peut imaginer que tant qu'elle s'entendra produire en dehors de cette norme, elle ne s'autorisera pas à agir avec la langue en toute circonstance, se privant ainsi de certaines possibilités de développer l'ensemble de ses habiletés. Mais à nouveau, des observables supplémentaires seraient nécessaires afin de déterminer à quel point cela la freine dans le développement de son répertoire langagier et, plus largement, dans son agir social en français.

\subsection{Dijana et les langues de la classe}

À d'autres moments, Dijana se positionne par rapport aux langues qu'elle pratique avec ses camarades d'études, comme ici :

Exemple 3

Di I speak e | English with Lotta | <hm hm> yeah I know! [e Lotta]

$\mathrm{Ma}$

[e j'ai pas dit] que c'était un problème

hein!

Di no it | it is! no I'm just saying what I feel | what I <ok! super alors! > | I think it's a problem $<$ why? $>$ because we should practice French I mean $<\mathrm{hm} \mathrm{hm}>$ and avec Pat- Patrizia parfois

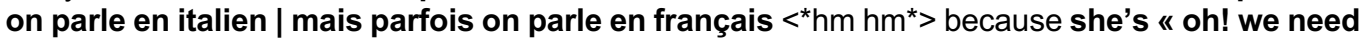
to practice french! » she's MORE | yeah | than Lotta | Lotta is not bothered to speak english I e XX speak french 
Ben Harrat, Malika et Anne-Christel Zeiter. « Derrière la langue, les positionnements sociaux. Pouvoir faire entendre sa voix en langue seconde ». Nouvelle Revue Synergies Canada, № 15 (2022)

$\mathrm{Ma}$

pourquoi tu penses?

Di no she just | <non mais je veux dire! > that's what she says $((\ldots))$ what SHE | what THEY say <ok> Lotta she's like «oh we know I'm not bothered to speak | French" ça dépend! <ok> it depends on the moment <*hm hm*> Patrizia | she's like "no we should | on doit parler français maintenant" on essaie de parler français mais | on CHANGE | mais ça va c'est | we | we | kind of fix it $<\mathrm{hm} \mathrm{hm}$ ! > and français | que | when we're all togheter

(Entretien Dijana 19.03.2019, 2'55'56-2'57'06, corpus Ben Harrat)

Ici, les positionnements de Dijana apparaissent en réseau, en fonction des relations entre ces trois amies âgées de 18 à 20 ans, et Dijana tend ${ }^{6}$, durant l'ensemble de l'entretien, à se positionner de manière similaire à ses deux camarades, donnant à voir le trio comme un tout: dans cet extrait, elle commence ainsi à s'autopositionner et à hétéropositionner Lotta comme locutrices anglophones lorsqu'elles interagissent ensemble. Mais l'intervention phatique de Ma en réponse à cette information représente un tournant réflexif. Dijana semble en effet la comprendre comme une marque de désapprobation, ce qui la conduit à se repositionner par un « ouais je sais » (« yeah I know ») marquant une opposition-concession : si elle valide la désapprobation qu'elle perçoit chez Ma en fonction de sa propre représentation des attentes de cette dernière quant à la langue qu'il faut parler entre camarades d'études en classe de langue (ici le français), elle oppose une justification de pourquoi elle ne le fait pas. Dans une succession d'auto- et d'hétérorepositionnements chevauchés à visée réparatrice, Dijana paraît accepter la réparation de Ma tout en se positionnant par rapport à son propre repositionnement (« ouais je sais »), manifestant une première fois dans cet extrait sa conscience de l'existence d'une tension entre les langues qu'elle est amenée à pratiquer. La représentation de Dijana sur l'intervention de l'enquêtrice reste toutefois formulée de la même manière, et elle continue à justifier ses pratiques langagières tout en les présentant comme un problème qu'elle et Lotta devraient éviter en parlant toujours français. Elle repositionne alors à nouveau l'enquêtrice comme une figure d'autorité universitaire représentant cette fois, en tant qu'enseignante de français, la norme du « bien apprendre la langue ${ }^{7}$.

Dijana présente ensuite ses interactions avec Patrizia comme des pratiques bilingues français/italien, qu'elle justifie par la narration des positionnements de Patrizia. Initiant une séquence narrative dans laquelle se succèdent cette fois-ci des positionnements de troisième ordre, Dijana hétéropositionne doublement Patrizia dans un rôle de garante de leur pratique du français en précisant que son amie lui rappelle qu'elles ont " besoin » de pratiquer cette langue et qu'elles la parlent donc ensemble par moments, au détriment de l'italien. La participante propose alors une comparaison sans équivoque entre ses deux amies : Patrizia est « PLUS » que Lotta, dont on apprend qu'elle « ne s'embête pas à parler [...| français » (notre traduction). Invitée à préciser ses propos, Dijana hésite d'abord (« non mais je veux dire! »), répondant finalement que ce sont les propos de Lotta elle-même. Poursuivant en ce sens, Dijana nous propose un hétéropositionnement de troisième ordre imitatif de Lotta, par le biais du discours rapporté, en précisant que leur choix d'une langue est contextuel. Elle hétéropositionne à nouveau Patrizia dans son rôle de rappel à l'ordre, un positionnement souvent performatif puisque, selon Dijana, les interventions de Patrizia auraient pour effet de les faire effectivement changer de langue. On pourrait alors imaginer que les « rappels à l'ordre " de Patrizia aient un effet positif sur leur acquisition du français, dans la mesure où elles s'orienteraient vers davantage de pratique, mais il faudrait avoir accès à cette partie de leurs pratiques réelles pour le savoir. Cette argumentation souligne toutefois que les trois amies font preuve de réflexivité quant à leur utilisation des différentes langues : si Dijana ne peut « cacher " sa tendance à bifurquer vers l'anglais - au quotidien comme lors de l'entretien - elle s'en dédouane par des positionnements de troisième ordre permettant à chaque membre du trio de faire entendre sa voix aux deux autres et à l'enquêtriceenseignante. Ceci permet à cette dernière de repérer un axe réflexif sur lequel s'appuyer dans une éventuelle future démarche didactique, une démarche qui toucherait à la qualité plurilingue du répertoire, à l'utilisation et à la valeur des langues dans différents contextes, et à l'importance d'en être conscient et de le marquer dans ses positionnements, en français ou non selon l'agir en cours.

\section{Pour conclure}

Analyser la voix des personnes en termes de positionnements énonciatifs liés à des représentations sociales permet ainsi d'identifier une grande complexité de relations de pouvoir, lesquelles structurent de manière évidente les pratiques sociales et langagières. Les deux cas présentés ici nous amènent à la situation paradoxale suivante : bien que manifestement capables de faire entendre leur voix en français, ce sont les représentations sociales de l'ensemble des participantes interrogées qui semblent freiner leur agir dans la langue. En effet, Nasrin semble justifier sa non-participation aux activités informelles avec ses collègues par une forte distinction prenant appui sur la différence de statut social qu'elle perçoit entre elle et les autres personnes, alors qu'à l'inverse, la proximité et la similarité que Dijana ressent avec ses amies, avec 
lesquelles elle échange quotidiennement dans différentes langues, semblent ne pas toujours l'encourager à agir pour s'approprier le français. Dans le cas de Dijana, il nous parait plus probable que l'importance accordée à la norme, et surtout l'écart qu'elle énonce entre sa prononciation et cette norme, constitue un frein dans sa participation à certains espaces sociaux. Enfin, on ne peut non plus exclure que lorsque le choix d'une langue ou d'une autre est possible entre interactant·e·s, celui-ci se détermine également en fonction de leur capacité - réelle comme perçue - à se positionner dans une langue ou dans une autre. La texture du répertoire langagier tel qu'il s'est construit par et dans les pratiques sociales antérieures ne permettent en effet pas de tout dire à tout le monde, dans n'importe quel contexte et n'importe quelle langue connue : le choix de la langue destinée à mener à bien certains agir spécifiques est alors crucial. On comprend mieux dès lors, et à la suite de Jeanneret (2010), l'enjeu d'une culture institutionnelle qui stimule la réflexivité des apprenants sur ces questions traitant à la fois d'insécurité linguistique (Remysen, 2018) et d'insécurité socio-langagière indexée à un agir particulier (Adami et André, 2010). Une démarche didactique qui amène les personnes apprenantes à modifier leurs représentations lorsque celles-ci les empêchent d'user de leur voix dans l'une ou l'autre des situations peut ainsi favoriser, selon nous, le cercle vertueux décrit par Jeanneret. En effet, ces moyens d'action complémentaires peuvent potentiellement rééquilibrer les échanges expérimentés et renforcer tant leur sentiment de compétence dans la langue d'appropriation que leur sentiment d'appartenance à la communauté des locuteurs francophones.

II s'agit néanmoins d'éviter de tomber dans le piège d"une vision « optimiste » et néolibérale de la voix agentive à laquelle les apprenant-e-s devraient théoriquement accéder. Si un travail sur les représentations et les positionnements des acteurs dans différents contextes permet d'amener les personnes à prendre conscience des rapports de pouvoir auxquels elles participent, ainsi qu'à réfléchir à des moyens concrets de rééquilibrer leurs échanges, les activités en classe ne peuvent couvrir qu'une infime partie des positionnements réellement vécus. Par ailleurs, les (re)positionnements souhaités peuvent également échouer à se déployer lorsque les interlocuteurs ou les contextes ne le permettent pas (Blackledge et Pavlenko, 2001). À ce titre, on peut alors aussi s'interroger sur nos propres représentations de spécialistes de l'acquisition des langues, qui varient par ailleurs d'une personne à l'autre au sein d'un même espace de formation, et de l'interférence potentielle de ces ambivalences avec les objectifs de nos apprenant·e·s. II importe ainsi notamment de réfléchir à comment les conjuguer avec celles de nos étudiant $\cdot e \cdot s$ lorsque nous les retrouvons en classe de langue. Le bénéfice collatéral serait, d'après nous, qu'enseignant·e·s et apprenant·e.s construisent et développent des voix plus communes que contraires afin d'éviter au maximum que ces confrontations de représentations ne contribuent à assourdir un peu plus les voix.

\section{Notes}

${ }^{1}$ Tous les prénoms utilisés dans l'article ont été anonymisés. L'exemple d'Afshin est tiré de l'enregistrement de tests oraux de langue dans une institution privée (corpus de Zeiter) et les autres du corpus de Ben Harrat.

${ }^{2}$ Voir https://www.unil.ch/files/live/sites/fle/files/shared/GReBL/Presentation GReBL 2013.pdf

${ }^{3}$ Nous traduisons.

${ }^{4}$ Les données mobilisées proviennent d'entretiens extraits d'un ensemble de quinze participant·e·s de l'étude de Ben Harrat.

${ }^{5}$ Ruzica et Irina sont deux autres participantes de l'étude de Ben Harrat, alors que Ming n'en fait pas partie. Cependant, Ming est une étudiante inscrite dans leur programme commun.

${ }^{6}$ Durant l'entretien, Dijana reçoit plusieurs messages de Lotta, qu'elle doit retrouver par la suite.

${ }^{7}$ Les notes de terrain de Ben Harrat indiquent que les représentations et les prescriptions des acteurs en matière de langues qu'il convient de parler peuvent changer d'un espace universitaire à un autre : autrement dit, les pratiques encouragées dans le cours $A$, ne sont ni celles prônées dans le cours $B$, ni celles que l'on peut rencontrer dans les divers espaces administratifs ou sociaux (cafétérias, bibliothèques, etc.) de l'institution. 
Ben Harrat, Malika et Anne-Christel Zeiter. « Derrière la langue, les positionnements sociaux. Pouvoir faire entendre sa voix en langue seconde ». Nouvelle Revue Synergies Canada, N 15 (2022)

\section{Conventions de transcription}

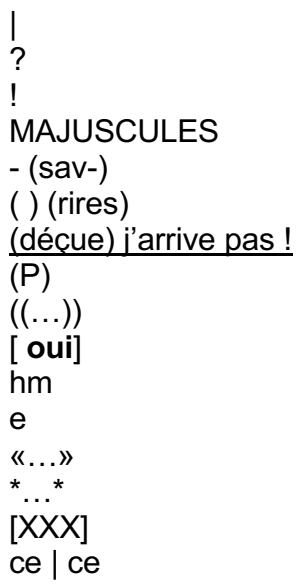

segmentation du discours

marqueur de questions

marqueur d'exclamations (surprise, émotion)

emphase

mot tronqué

commentaires de la transcriptrice utiles pour la contextualisation

soulignement qui marque une émotion dans le discours

pause significative (de plus de deux secondes)

coupure au sein d'un extrait

chevauchement

marqueur d'approbations

marqueur d'hésitations

représentation du discours autre

chuchotement

syllabe inaudible

répétition 
Ben Harrat, Malika et Anne-Christel Zeiter. « Derrière la langue, les positionnements sociaux. Pouvoir faire entendre sa voix en langue seconde ». Nouvelle Revue Synergies Canada, Nº 15 (2022)

\section{Bibliographie}

Adami, H. et André, V. (2010). Les migrants en insécurité linguistique au travail : analyses et perspectives. Points communs, 40, 1-5.

Ben Harrat, M. L'appropriation langagière en milieu universitaire. Élaborer ses représentations et ses positionnements sociaux pour (re)trouver sa voix(e). Corpus d'une thèse de doctorat (en cours), Université de Lausanne, Faculté des Lettres.

Blackledge, A. et Pavlenko, A. (2001). Negotiation of identities in multilingual contexts. International Journal of Bilingualism, 5(3), 243-257.

Blommaert, J. et Backus, A. (2012). Superdiverse Repertoires and the Individual. Tilburg Papers in Culture Studies, 24, 1-33.

Bourdieu, P. (2001). Langage et pouvoir symbolique. Seuil Essais.

Busch, B. (2013). Mehrsprachigkeit. Facultas Verlag UTB.

Busch, B. (2015). Expanding the notion of the linguistic repertoire: On the concept of Spracherleben - The lived experience of language. Applied Linguistics, 38(3), 1-20. https://doi.org/10.1093/applin/amv030

Davies, B. et Harré, R. (1990). Positioning: The discursive production of selves. Journal for the Theory Of Social Behaviour, 20 (1), 43-63.

Dortier, J.-F. (2008). Représentation sociale. Dictionnaire des sciences humaines. Sciences humaines Éditions, 633-634.

Dubar, C. (2010). La socialisation : Construction des identités sociales et professionnelles. Armand Colin.

Duff, P. A. (2003). New directions in second language socialization research. Korean Journal of English Language and Linguistics, 3, 309-339.

Foucault, M. (1970). L'Ordre du discours. Gallimard, 2015.

Grize, J.-B. (1990). Logique et langage. Ophrys.

Harré, R. (2012). Positioning theory: Moral dimensions of social-cultural psychology. Dans J. Valsiner (dir.). The Oxford Handbook of Culture and Psychology (p. 191-206), Oxford University, 191-206.

Jeanneret, T. (2010). Trajectoires d'appropriation langagière et travail identitaire : Données et analyses. Bulletin suisse de linguistique appliquée, 1(1), 27-45.

Jodelet, D. (1989) Représentations sociales : Un domaine en expansion. Dans D. Jodelet (dir.), Les représentations sociales (p. 47-78), PUF.

Jodelet, D. (2005). Formes et figures de l'altérité. Dans M. Sanchez-Mazas et L. Licata (éds.), L'Autre : Regards Psychosociaux (p. 23-47). Les Presses de l'Université de Grenoble.

Jodelet, D. (2008). Le mouvement de retour vers le sujet et l'approche des représentations sociales. ERES Connexions, 89(1), 25-46.

Kayi-Aydar, H. (2019). Positioning Theory in Applied Linguistics. Research Design and Applications. Palgrave MacMillan.

Remysen, W. (2018). L'insécurité linguistique à l'école : Un sujet d'étude et un champ d'intervention pour les sociolinguistes. Dans N. Vincent et S. Piron (dir.), La Linguistique et le Dictionnaire au Service de l'Enseignement du Français au Québec (p. 25-59). Nota Bene.

Roberts, C. (1999). Acquisition des langues ou socialisation dans et par le discours? Langages, 134, 101123. 
Ben Harrat, Malika et Anne-Christel Zeiter. « Derrière la langue, les positionnements sociaux. Pouvoir faire entendre sa voix en langue seconde ». Nouvelle Revue Synergies Canada, Nº 15 (2022)

Van Langenhove, L. et Harré, R. (1999). Introducing Positioning theory. Dans L. Van Langenhove et R. Harré (dir.), Positioning Theory (p. 14-31). Blackwell.

Wenger, E. (2009). La théorie des communautés de pratique. Apprentissage, sens et identité. Les Presses de l'Université de Laval.

Zeiter, A.-C. (2018). Dans la langue de l'autre. Se construire en couple linguistiquement mixte. ENS-Lyon.

Zeiter, A.-C. (2019). Déconstruire la motivation pour rencontrer le désir (de langue). Revue TDFLE [En ligne]. http://revue-tdfle.fr/actes-1-44/168-deconstruire-la-motivation-pour-rencontrer-le-desir-de-langue- 